\title{
Análisis del sentido de comunidad como promotor de la resiliencia comunitaria en habitantes de ciudades en riesgo de desastres naturales
}

\section{Analysis of the sense of community as a promoter of community resilience in city dwellers at risk of natural disasters}

\section{Mónica Tatiana Perdomo Pérez ${ }^{1}$ \\ Carlos Reyes Valenzuela? \\ Flor Sánchez ${ }^{3}$}

( Recepción: 16/09/2019

(-) Aprobación: 18/10/2019

( Publicación: 19/12/2019

\section{Para citar este artículo:}

Perdomo Pérez, M. T., Reyes Valenzuela, C., \& Sánchez, F. (2019). Análisis del sentido de comunidad como promotor de la resiliencia comunitaria en habitantes de ciudades en riesgo de desastres naturales. Indagare, (7), 14-18. https://doi.org/10.35707/indagare/702

\section{(6) $(\mathbb{\theta} \Theta \Theta$}

1Profesora del Programa de Psicología. Universidad de Ibagué. ORCID: 0000-0002-1423-1366. tatiana.perdomo@ unibague.edu.co

${ }^{2}$ Coordinador del Programa Andino de Derechos Humanos. Universidad Andina Simón Bolívar, sede Ecuador. ORCID: 0000-0001-8911-700X.

${ }^{3}$ Profesora titular de la Facultad de Psicología. Universidad de Autónoma de Madrid. ORCID: 0000-0002-8058-5584. 


\title{
Resumen
}

Esta investigación busca conocer el nivel de sentido de comunidad, además de procesos socioemocionales, con los que cuentan los ciudadanos de la ciudad de Ibagué para hacer frente a situaciones traumáticas como lo es la experiencia de vivir algún tipo de desastre natural, teniendo en cuenta los numerosos riesgos a los que se está expuesto. Para tal fin, se contó con el reporte de cuatrocientos habitantes de la ciudad; la recolección de información se realizó con el apoyo de la Dirección de Juventudes y Emprendimiento de la Alcaldía de Ibagué. Los principales resultados se orientan a identificar una alta percepción de riesgo de ser víctima de terremotos e inundaciones, un moderado nivel de sentido de comunidad, participación cívica y de procesos socioemocionales comunitarios.

\begin{abstract}
This research seeks to know the level of sense of community, as well as social-emotional processes, that citizens of the city of Ibagué have to face traumatic situations such as the experience of living some kind of natural disaster, bearing in mind numerous risks to which they are exposed to. For that purpose, a report was carried out for four hundred inhabitants of the city; data collection was accomplished with the support of the Directorate of Youth and Entrepreneurship of the Municipality of Ibagué. The main outcomes are aimed at identifying a high perception of risk of being a victim of earthquakes and floods, a moderate level of sense of community, civic participation and social-emotional community processes.
\end{abstract}

Palabras claves: Desastres naturales, sentido de comunidad, riesgo, amenazas.

Key words: Natural disasters, sense of community, risk, hazards.

\section{Introducción}

Aunque es cierto que una de las principales consecuencias que deja la ocurrencia de un desastre natural es la muerte de personas y la pérdida de bienes materiales, no se puede dejar de lado el alto contenido traumático que para los sobrevivientes trae el vivir una experiencia de riesgo. Por tal motivo, es fundamental que las comunidades en riesgo de desastres naturales cuenten con herramientas sociales y psicológicas que les permitan hacer frente a la experiencia y poder seguir adelante. 
Es, pues, importante identificar procesos psicosociales, como el sentido de comunidad, porque pueden ser fuente de procesos más adaptativos para las personas que sobreviven a un desastre natural, permitiendo comprender cómo y a quienes afecta psicológicamente este tipo de experiencias, abriendo espacio a intervenciones psicosociales oportunas y efectivas.

\section{Método}

Este proyecto de investigación se basa en un diseño mixto de alcance descriptivoexploratorio, visible en las estrategias de recolección, análisis de datos e interpretación de estos. Participaron del estudio 400 habitantes de la ciudad de Ibagué, 216 hombres y 184 mujeres, distribuidos en barrios de las 13 comunas de la ciudad de Ibagué.

El trabajo de campo consistió en la recolección puerta a puerta de la información a través de un cuestionario, que preguntaba por la percepción de riesgo, tipo de participación cívica, sentido de comunidad, apego e identidad al lugar. La recolección de información contó con el apoyo de los Gestores de Paz, quienes conocían el territorio. Además, estudiantes y el equipo de investigación de Unibagué. Esta fase de levantamiento de datos cuantitativa duró aproximadamente dos meses. Complementario a este ejercicio se convocaron siete grupos focales en diferentes sectores de la ciudad, que buscaban conocer la forma en que habían construido sus recursos comunitarios. Para finalmente analizar todos los datos, en etapa que se encuentra en su recta final.

\section{Resultados}

Los resultados de esta investigación indican que el sentido de comunidad de los habitantes de la ciudad de Ibagué se presenta de manera moderada. Esta medida puede hacer suponer que los lazos de confianza y de trabajo comunitario son débiles y que, posiblemente ante una emergencia natural, los habitantes de la ciudad respondan de manera individualista y poco articulada.

En las dimensiones socioemocionales, tales como identidad y apego al lugar, los resultados son similares y alineados a los del sentido de comunidad, evidenciando bajo arraigo e identificación con su territorio más próximo, como es el barrio, y uno macro como lo es la ciudad. Lo que puede indicar bajo sentido de pertenencia y, posiblemente, una escasa disposición para generar compromisos significativos para preservar su territorio, los valores y costumbres de ese espacio. Se encontró que la participación cívica de los habitantes de la ciudad es limitada. Los espacios que más se frecuentan son los asociados con eventos religiosos y con actividades deportivas. 
Los habitantes de la ciudad presentan percepción de riesgo alta ante desastres naturales asociados con terremotos e inundaciones. Sin embargo, se encontró con asombro que los habitantes no se perciben en riesgo de deslizamiento o remoción de masa, a pesar de ser el evento natural que se presenta con mayor frecuencia en la ciudad.

\section{Potencial uso}

Se espera que los resultados de este estudio sirvan de insumo para (1) incidir en acciones institucionales que faciliten que las comunidades estén mejor preparadas ante una posible emergencia. (2) Diseño de propuestas de intervención comunitaria, orientadas a responder a las necesidades que esta línea de base permite identificar, principalmente en lo que respecta a incrementar el sentido de comunidad en los barrios de la ciudad.

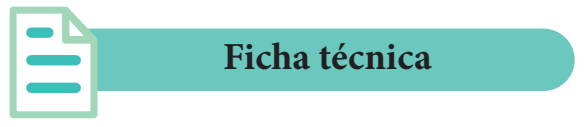

Título del proyecto:

Análisis del sentido de comunidad como promotor de la resiliencia comunitaria en habitantes de ciudades en riesgo de desastres naturales.

PRIT: Educación y bienestar para el desarrollo humano integral.

Código del proyecto: $18-553-\mathrm{INT}$.

Palabras claves: Desastres naturales, sentido de comunidad, riesgo, amenazas.

Grupo de investigación: Educación, salud y sociedad, GESS.

Investigador principal: Mónica Tatiana Perdomo Pérez.

\section{Referencias}

Berroeta, H., Ramoneda, A., Rodríguez, V., DiMasso, A., \& Vidal, T. (2015). Apego de lugar, identidad del lugar, sentido de comunidad y participación cívica en personas desplazadas de la ciudad de Chaitén. Magallania, 43(3), 51-63. http://dx.doi.org/10.4067/S0718-22442015000300005

Hombrados, M. (2011). Sentido de comunidad. En Fernández, I., Morales, J. \& Molero, F. (Coord.). Psicología de la intervención comunitaria, (pp. 97-126). Madrid: Desclée de Brower DDB.

Sánchez, A. (2009). Validación discriminante de una escala de sentimiento de comunidad: análisis comparativo de dos comunidades. International Journal of Psychology and Psychological Therapy, 9 (2), 161-176. Recuperado de https://www.ijpsy.com/volumen9/num2/229.html 